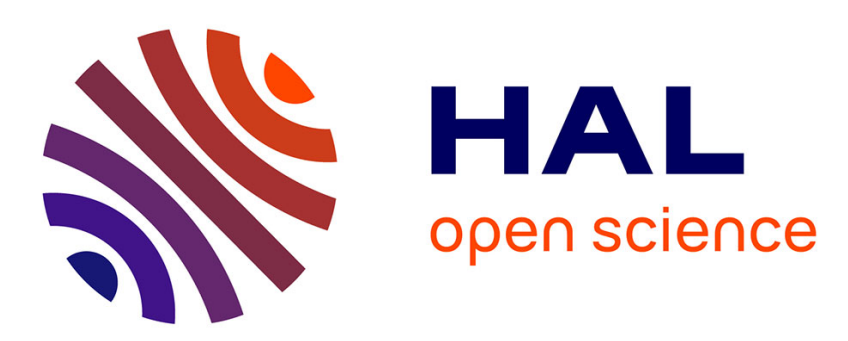

\title{
Divided medium-based model for analyzing the dynamic reorganization of the cytoskeleton during cell deformation
}

Jean-Louis Milan, Sylvie Wendling-Mansuy, Michel Jean, Patrick Chabrand

\section{- To cite this version:}

Jean-Louis Milan, Sylvie Wendling-Mansuy, Michel Jean, Patrick Chabrand. Divided medium-based model for analyzing the dynamic reorganization of the cytoskeleton during cell deformation. Biomechanics and Modeling in Mechanobiology, 2007, 6 (6), pp.373-390. 10.1007/s10237-006-0057-7 . hal00475545

\section{HAL Id: hal-00475545 \\ https://hal.science/hal-00475545}

Submitted on 15 Nov 2017

HAL is a multi-disciplinary open access archive for the deposit and dissemination of scientific research documents, whether they are published or not. The documents may come from teaching and research institutions in France or abroad, or from public or private research centers.
L'archive ouverte pluridisciplinaire HAL, est destinée au dépôt et à la diffusion de documents scientifiques de niveau recherche, publiés ou non, émanant des établissements d'enseignement et de recherche français ou étrangers, des laboratoires publics ou privés. 


\title{
Divided medium-based model for analyzing the dynamic reorganization of the cytoskeleton during cell deformation
}

\author{
J. L. Milan · S. Wendling-Mansuy • M. Jean • \\ P. Chabrand
}

Received: 3 February 2006 / Accepted: 7 September 2006 / Published online: 25 October 2006

(C) Springer-Verlag 2006

\begin{abstract}
Cell deformability and mechanical responses of living cells depend closely on the dynamic changes in the structural architecture of the cytoskeleton (CSK). To describe the dynamic reorganization and the heterogeneity of the prestressed multi-modular CSK, we developed a two-dimensional model for the CSK which was taken to be a system of tension and compression interactions between the nodes in a divided medium. The model gives the dynamic reorganization of the CSK consisting of fast changes in connectivity between nodes during medium deformation and the resulting mechanical behavior is consistent with the strain-hardening and prestress-induced stiffening observed in cells in vitro. In addition, the interaction force networks which occur and balance to each other in the model can serve to identify the main CSK substructures: cortex, stress fibers, intermediate filaments, microfilaments, microtubules and focal adhesions. Removing any of these substructures results in a loss of integrity in the model and a decrease in the prestress and stiffness, and suggests that the CSK substructures are highly interdependent. The present model may therefore provide a useful tool for understanding the cellular processes involving CSK reorganization, such as mechanotransduction, migration and adhesion processes.
\end{abstract}

J. L. Milan · S. Wendling-Mansuy ( $ه) \cdot$ P. Chabrand CNRS-USR 2164 Laboratoire d'Aérodynamique et de Biomécanique du Mouvement, Parc Scientifique et Technologique de Luminy, 13288 Marseille Cedex 9, France e-mail: sylvie.wendling@univmed.fr

M. Jean

CNRS-UPR 7051 Laboratoire de Mécanique et d'Acoustique, 13451 Marseille Cedex 20, France
Keywords Mechanical cell responses . Multicompartment · Stress-strain relationships . Stiffness

\section{Introduction}

Many in vitro and in vivo studies have shown that the cytoskeleton (CSK) plays a fundamental role in the mechanisms underlying cell processes such as growth, differentiation, proliferation, motility and protein synthesis (Chicurel et al. 1998; Thoumine and Ott 1996; Davies et al. 1994; Planus et al. 1999; Hughes-Fulford 2004). Cell deformability and mechanical responses depend basically on several interactive processes between factors such as the structural CSK-architecture and cell/cell or cell/extracellular matrix attachment. The mechanical forces exerted through the extracellular matrix are balanced by internal forces exerted by the cytoskeleton. This force balance is known to be mediated by changes in the internal CSK structure, an interconnected biopolymer system embedded in the cytoplasm. The CSK supports the entire cell and links the nucleus to the surface adhesion receptors, involving a hierarchically organized multi-modular structure ranging from the molecular to the overall cellular scale (Maniotis et al. 1997; Ingber 2000; Pienta and Coffey 1991; Wang 1998). The complex mechanisms whereby the CSK mediates force transmission, and the contribution of each of its substructures to the structural stability of the cell have not yet been clearly elucidated.

Several structural models have been proposed to describe the mechanical behavior of cells in terms of the characteristic properties of the cytoskeleton components (Stamenovic et al. 1996; McGarry and Prendergast 2004; 
Volokh et al. 2000; Coughlin and Stamenović 2003; Satcher and Dewey 1996; Wendling et al. 1999). For instance, structural models based on the tensegrity concept have been developed, which mimic several aspects of the mechanical behavior of adherent cells such as strain-hardening (Wang et al. 1993), prestress-induced stiffening (Wang et al. 2002; Pourati et al. 1998; Wendling et al. 2003) and the viscoelastic properties (Canadas et al. 2002, 2006; Sultan et al. 2004). The generalization of some characteristic mechanical laws governing spherical tensegrity structures has also led to identifying two compartments of the CSK in twisted adherent cells (Wendling et al. 2003; Laurent et al. 2002) resulting from two different arrangements of actin filaments: the smooth submembrane cortical network, and the strongly contractile actin bundles or stress fibers (SFs) which extend between focal adhesion points. These two CSK components were found to be prestressed and to show a stress-hardening behavior (Wendling et al. 2003; Laurent et al. 2002). However, in these studies, the cortical network and stress fiber network were dealt with separately on two different subcellular scales so that it was not possible to relate the local and global mechanical properties to the changes of the overall cellular stiffness. McGarry and Prendergast (2004) recently proposed a finite element model including prestressed cytoskeleton, cytoplasm, nucleus and membrane components. This model based on a continuum medium and a tensegrity system describes the nonlinear structural behavior of an adherent cell in terms of variable compliance along the cell surface and changes in stiffness during the cell spreading. This approach can be used to analyze the contribution of each structural component to the stability and stiffness of the cell. However, it fails to reflect the increase in the stiffness which occurs when the cell is spreading over the substrate, as observed by Thoumine et al. (1999) who suggested that the reorganization of the cytoskeleton may contribute importantly to the stiffening of the cell during deformation.

The dynamic rearrangement of the CSK which occurs during cell deformation is not taken into account in either McGarry's model or the tensegrity models. The authors of various studies have described the dynamic behavior of the CSK network, which constantly rearranges itself via polymerization and depolymerization processes (Ingber 1997; Harris et al. 1980; Kaverina et al. 1998; Mooney et al.1995). The length, number and crosslinking of the cytoskeletal filaments are all dynamically regulated by the cell (Heidemann and Wirtz 2004). As the microfilaments (MFs) and microtubules (MTs) can alternately develop and disappear, the interconnections are very variable. Via these spatial rearrangements, MFs and MTs can generate the intracellular forces required to strengthen or loosen the CSK network locally, in order to balance the external forces applied via the focal adhesions sites (Heidemann et al. 1999; Chicurel et al. 1998).

To describe the dynamic reorganization of the hierarchical prestressed CSK structure of adherent cells, it was proposed in the present study to use a novel approach based on divided medium theory. The CSK Divided Medium model (or CDM model) that we developed consists of a set of inner and boundary nodes surrounding a rigid nucleus, forming the "divided medium" and interacting together via compressive and tensile forces. The resulting networks of compressive and tensile forces within the medium are assumed to model the various filamentous lattices of the CSK. Since, the divided medium subjected to these interaction forces is at equilibrium, any change in the external stress will affect the system of interacting forces and give the divided medium a new equilibrium configuration.

Here we describe the laws governing the interaction forces at work in the divided medium and the method used to compute equilibrium configurations. Using this CDM model, it was then attempted to assess the contributions of several CSK substructures, namely the stress fiber network, actin cortex, MF, MT and intermediate filament (IF) networks as well as the focal adhesion points, to the stability and the mechanical responses of adherent cells under quasi static compression/stretching loading conditions. This model provides a new means of investigating the forces transmitted from the membrane to the nucleus.

\section{Methods}

The CDM model forms an $80-\mu \mathrm{m}$ diameter half-disk, representing a cell adhering to a flat, rigid substrate in two dimensions. The medium of the CDM model is constituted by a set of nodes arbitrarily placed around a $20-\mu \mathrm{m}$ diameter floating rigid disk simulating the nucleus. The CSK of the adherent cell is modeled in the form of a network of tension forces balanced by a network of compression forces. These forces are exerted between nodes in the divided medium constituting the model. Some given elastic tensile forces are exerted between specific remote nodes, while constant tensile forces and compressive forces are exerted between neighboring nodes. The distance between two nodes therefore cannot be smaller than a given threshold value. This is a unilateral condition which is applied by adding a compressive force between the nodes if necessary. From the mechanical point of view, this law governing the interactions between neighboring nodes may be 


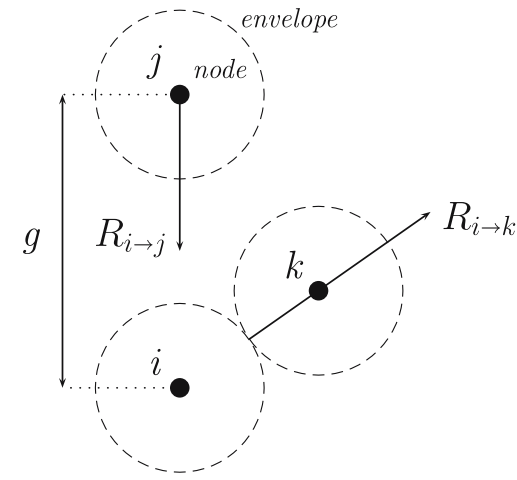

Fig. 1 Description of interactions between the nodes and their associated envelopes. $g$ denotes the gap between the nodes $i$ and $j, R_{i \rightarrow j}$ the radial reaction force exerted by $i$ on $j, R_{i \rightarrow k}$ the radial reaction force exerted by $i$ on $k . R_{i \rightarrow j}$ stands here for an distant tension force resulting from the Elastic Wire interaction law, and $R_{i \rightarrow k}$ for a compression force resulting from the Cohesive Contact interaction law

considered as a contact law between two virtual circular and rigid envelopes centered on the nodes and satisfying impenetrability conditions (see Fig. 1).

\subsection{Constitutive equations based on divided medium theory}

The mechanical behavior of the CDM model is characterized by both the equations of interaction between the constitutive nodes and the dynamic equation for the whole set of nodes. The interaction between two nodes forming a pair labelled by the index $\alpha$ is described on the local scale in terms of the distance between them which is called the gap $g^{\alpha}$, their relative velocity which is denoted $\mathbf{U}^{\alpha}$, and the radial reaction force $\mathbf{R}^{\alpha}$ which is the force exerted by one node on the other (see Fig. 1). The interaction between these two nodes is determined by relation which will be presented below, and is referred to as:

$$
I\left(g^{\alpha}, \mathbf{R}^{\alpha}\right)
$$

The dynamic Lagrange equation is:

$$
\mathbf{M} \ddot{\mathbf{q}}=\mathbf{F}(t)+\mathbf{r}
$$

The components of $\mathbf{q}$ are the coordinates of all the nodes. $\mathbf{q} \in \mathbb{R}^{N}$, where $N$ is the degrees of freedom of the system; $N=2 n$, where $n$ is the number of nodes. The components of $\mathbf{r}\left(\mathbf{r} \in \mathbb{R}^{N}\right)$ are the resulting forces applied to the nodes. $\mathbf{M}$ is the mass matrix of the system: it is a diagonal matrix, and its components are the node masses. $\mathbf{F}(t)$ stands for the explicitly known excitation forces. The problem in hand is assumed to be a quasi- static problem. However, in the model, the constitutive nodes are taken to be free nodes although they interact with each other. Equation (2) ruling the evolution of the system is therefore a dynamic equation.

Variables $\dot{\mathbf{q}}$ and $\mathbf{U}^{\alpha}, \mathbf{r}$ and $\mathbf{R}^{\alpha}$ are related by the linear mapping $\mathbf{B}^{\alpha}$ and its transposed form, $\mathbf{B}^{* \alpha}$ as follows:

$$
\left\{\begin{array}{l}
\mathbf{U}^{\alpha}=\mathbf{B}^{\alpha}(\mathbf{q}) \dot{\mathbf{q}} \\
\mathbf{r}=\sum_{\alpha} \mathbf{B}^{* \alpha}(\mathbf{q}) \mathbf{R}^{\alpha}
\end{array}\right.
$$

For instance, in the case of a pair of nodes $\alpha$, the relative velocity $\mathbf{U}^{\alpha}$ between the two nodes is the difference between the velocity of the nodes; if $\mathbf{R}^{\alpha}$ is the reaction between the nodes, $\mathbf{r}$ will result from the pair of forces $\left(+\mathbf{R}^{\alpha},-\mathbf{R}^{\alpha}\right)$ exerted on each node. The gap $g^{\alpha}$ between the pair of nodes $\alpha$ is related to $U_{N}^{\alpha}$, the normal component of the relative velocity $\mathbf{U}^{\alpha}$, is as follows:

$\dot{g}^{\alpha}=U_{N}^{\alpha}$

Equations (1)-(4) along with the initial and boundary conditions completely define the constitutive equations in the CDM model.

2.2 Laws of interaction between the constitutive nodes in the medium

To describe the spatial architecture of the cytoskeleton taking into account its various known components (the cortical actin network, stress fiber network, microtubule network, intermediate filament network, nucleusbinding complexes and focal adhesion points), several node species associated with specific interaction laws are defined in the model. The node interactions (1) are governed by laws of two kinds which are either directly defined node-to-node or involve the surrounding rigid envelopes.

The first law which we have called Cohesive Contact law, is given by the set of equations (5) and shown in Fig. 2a. This law describes contact interactions between the virtually rigid envelopes surrounding the nodes and generates compression or tension forces between neighboring nodes, which we have called close compressions and close tensions (see Fig. 1). It is based on the classical Signorini and Lennard-Jones models (Jean 1999).

$$
\left\{\begin{array}{l}
g=g_{i} \Rightarrow R>-c \\
\left.g \in] g_{i}, g_{c}\right] \Rightarrow R=-c \\
g>g_{c} \Rightarrow R=0 \\
g_{c}=g_{i}+a
\end{array}\right.
$$

In Cohesive Contact, compression force $R>0$ occurs between two interacting nodes so as to ensure 

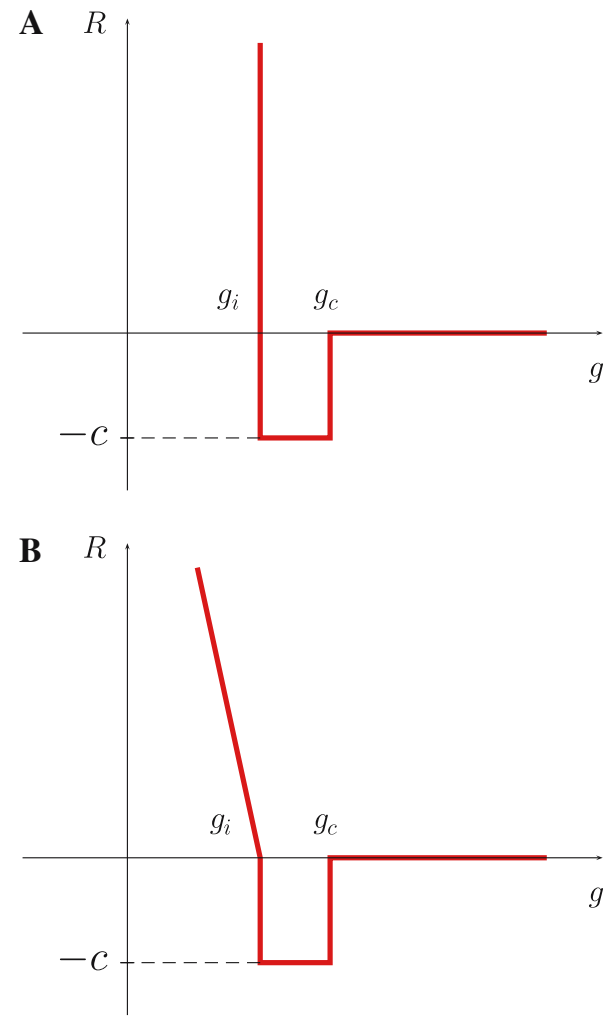

Fig. 2 a Cohesive Contact interaction law. This law defines the relationship between the normal reaction $R$ and the gap $g$ between neighboring nodes in involving their surrounding envelopes. These envelopes are rigid and undergo compression force $R>0$ while keeping a minimum gap $g_{i}$ between the nodes. In a small range of gaps, $\left[g_{i} ; g_{c}\right]$, nodes are subjected to a tension force $R<0$ due to the cohesion $c$ between envelopes. b Soft Cohesive Contact interaction law. Like the Cohesive Contact law, this law defines the relationship between the normal reaction $R$ and the gap $g$ between neighboring nodes but deals with compressive interactions by introducing penetrability $g<g_{i}$ between the node envelopes and an elastic compressive $R>0$ that prevents penetration. In a small range of gaps, $\left[g_{i} ; g_{c}\right]$, nodes are subjected to a tension force $R<0$ due to the cohesion $c$ between the envelopes

impenetrability between their surrounding rigid envelopes. The gap $g$ between two nodes cannot therefore be smaller than $g_{i}$, which is the sum of the radiuses of their envelopes. Within the 80- $\mu \mathrm{m}$ diameter medium, the minimum gap $g_{i}$ between nodes is around $1 \mu \mathrm{m}$. Besides, in this law, tension force $R<0$ occurs between nodes due to the constant cohesion tension $c$ existing between their envelopes in contact. This cohesion prevents the nodes from separating until a threshold distance $a$ (corresponding to distance $g_{c}$ between nodes) is reached between envelopes, above which the contact is broken. The product (c.a) gives the energy required to completely separate the two envelopes.

To analyze the effects of the local compressive rigidity on the overall stiffness of the model, an alternative form of the Cohesive Contact law, called the Soft Cohesive

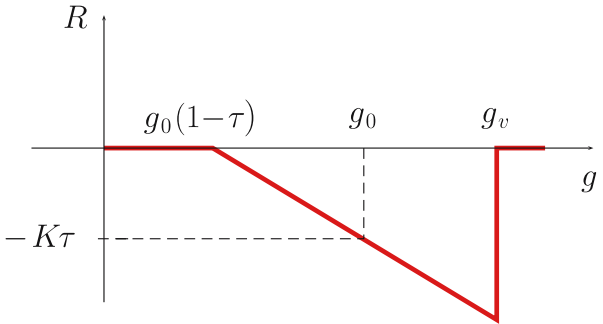

Fig. 3 Elastic Wire interaction law. This law introduces a tensile force $R \leq 0$ between remote nodes, which increases linearly with the gap $g$ and with a given rigidity $K$ up to a threshold distance $g_{v}$. The prestrain $\tau$ in the Elastic Wire interaction means that an effective tension is transmitted between nodes at the beginning of the simulation when the nodes are apart $g_{0}$

Contact law, which is given by the set of equations (6) and shown in Fig. 2b, introduces softer compressive interactions between neighboring nodes, making interpenetration between the virtually rigid envelopes possible.

$$
\left\{\begin{array}{l}
g \leq g_{i} \Rightarrow R=S .\left(g_{i}-g\right) \\
\left.g \in] g_{i}, g_{c}\right] \Rightarrow R=-c \\
g>g_{c} \Rightarrow R=0 \\
g_{c}=g_{i}+a
\end{array}\right.
$$

As the node envelopes interpenetrate, the compressive force $R>0$ increases linearly with the penetration $\left(g_{i}-g\right)$ according to a compressive rigidity written $S>0$.

The second law, which is called the Elastic Wire law, is used to generate distant tensile forces, between specialized remote nodes. This law acts like a virtual prestrained elastic wire. The tension is proportional to the stretching and becomes null when the elastic wire is loose. As written in the set of equations (7) and shown in Fig. 3, the tension $R$ between two nodes is given as a function of the gap $g$, where $g_{0}$ is the gap at the beginning of the simulation, taking $K>0$ to denote the rigidity and $\tau>0$ to denote the prestrain in the virtual elastic wire.

$\left\{\begin{array}{l}g \geq 0 ; R \leq 0 \\ R=-K\left(\frac{\Delta g}{g_{0}}+\tau\right) \\ g \geq g_{v} \Rightarrow R=0\end{array}\right.$

The gap $g_{0}$ between interacting nodes is deduced from the coordinates of the nodes at the beginning of the simulation. The rigidity $K$ is defined as a force $F$ per normalized change in the size of the gap:

$K=F / \frac{\Delta g}{g_{0}}$

The prestrain $\tau$ introduces into this law a non-zero tension between the two nodes. If the length of the wire is 
greater than the distance $g_{v}$, the Elastic Wire interaction ceases.

\subsection{Various node species in the medium for modelling the CSK components}

To account for the various CSK components, the nodes that constitute the medium in the CDM model are defined in terms of distinct node species, to which specific interaction laws apply. These interaction laws are defined from the Cohesive Contact and Elastic Wire laws with different values of the parameters $\left(c, a, S, K, \tau, g_{v}\right)$. Some of the parameters are estimated based on experimental data obtained on the mechanical properties of specific CSK components, while the others are adapted so as to ensure the mechanical integrity of the model. For instance, to account for the focal adhesion points of adherent cells, some nodes called the focal adhesion nodes, are distinguished at the boundary (on the apical and the basal sides) of the medium (see Fig. 4a). These nodes interact with the external nodes in the substrate via their surrounding envelopes in line with the Cohesive Contact law, with a very high cohesive tension value $c=150 \mathrm{nN}$ at a maximum distance $a=2 \mu \mathrm{m}$. The focal adhesions nodes located on the apical side, which is devoid of external contacts, are inactive and constitute precursors of possible adhesion points.

In cells, focal adhesion points exert tension forces of $[10-70] \mathrm{nN}$ on the substrate with focal adhesion areas of [1-10] $\mu \mathrm{m}^{2}$ (Balaban et al. 2001; Tan et al. 2003). In agreement with these biological data, the tension force generated between a single focal adhesion node with a $2-\mu \mathrm{m}$ diameter adhesion area and the substrate was found to average $30 \mathrm{nN}$. In addition, the focal adhesion nodes interact according to specific Cohesive Contact laws $\left(c=100 \mathrm{nN}, a=1 \times 10^{-1} \mu \mathrm{m}\right)$ with the other nodes on the boundary.

The CSK tensile networks of SFs and IFs, which extend through the whole cell, are simulated in the CDM model by the so-called distant tension network (TN) generated by the Elastic Wire laws between the focal adhesion nodes, the inner nodes of the perinuclear region called perinuclear nodes, plus several points on the nuclear boundary, as shown in the Fig. 4 a.

In the TN network, the distinction is made between the perinuclear tensile network formed between the perinuclear nodes and the nuclear boundary and the large tensile network formed between the focal adhesion nodes and the perinuclear nodes. The perinuclear tensile network simulates the IF network, which is connected to the nucleus and is particularly prominent in the perinuclear region in the cell. The large tensile network, which consists of the IF network extended to the periphery
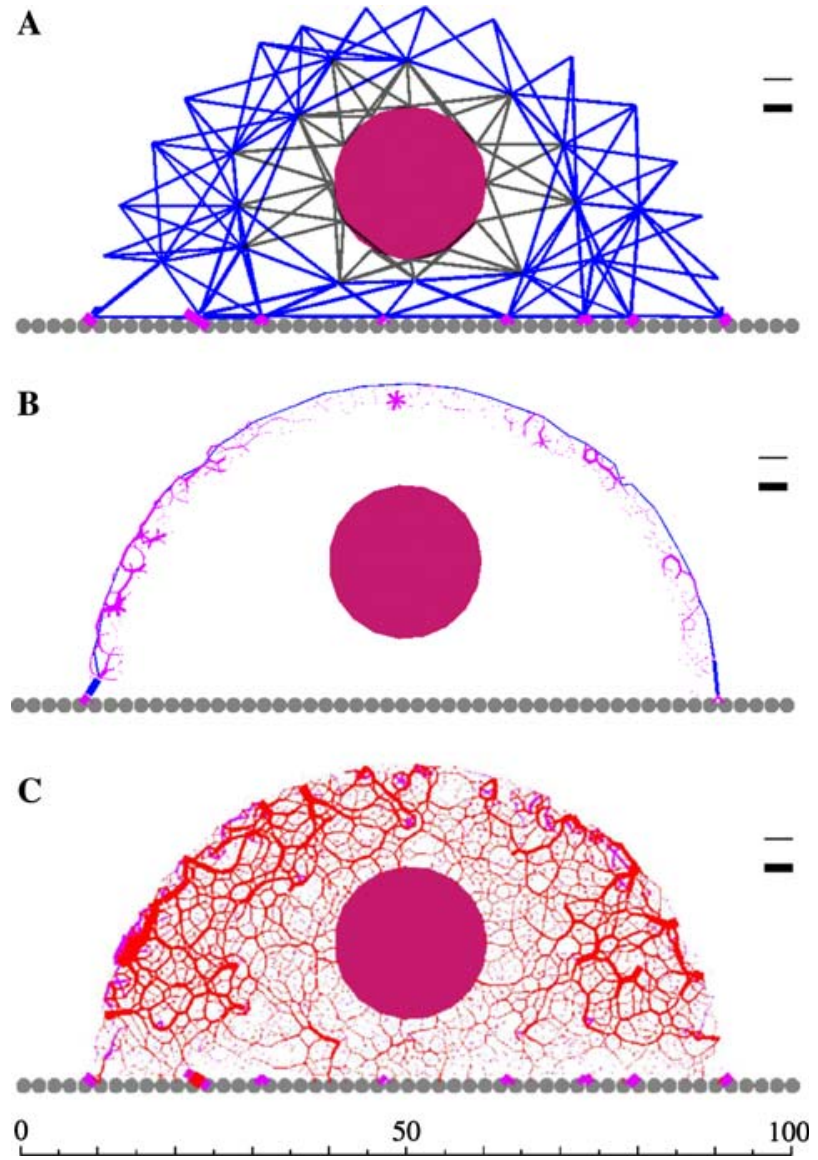

Fig. 4 Tension and compression interactions between nodes in the divided medium in the reference state. Forces are displayed in segments which connect the nodes during interactions and the thickness of these segments is proportional to the magnitude of forces; the two reference bars correspond to magnitudes of 10 and $70 \mathrm{nN}$. The unit of length is micrometer. Blue lines give distant tension forces. Gray lines around the nucleus give distant tension forces which are null in the reference state. Red segments stand for close compressive forces and purple ones stand for close tensile forces. Node envelopes are not shown here. a Distant tension network connected to the substrate via cohesive contact forces. b Close tension network associated to a distant tension force on the cortical side. c Close compression and tension networks involving the whole set of nodes

of the cell, mainly includes the SF network, which is assumed to be located at the basal and distal regions of the cell.

The Elastic Wire law, which governs the large distant tension network simulating the stress fiber network, is defined (rigidity $K=50 \mathrm{nN}$, prestrain $\tau=20 \%$, threshold distance $g_{v}=20 \mu \mathrm{m}$ ) in line with experimental data published by Deguchi et al. (2005). These authors obtained a rigidity (i.e. force/strain ratio) of $45.7 \mathrm{nN}$ and a $20 \%$ prestrain in SFs under physiological conditions (Deguchi et al. 2005).

The Elastic Wire law of the perinuclear distant tension network characterizing the IF network is defined 
(rigidity $K=150 \mathrm{nN}$, prestrain $\tau=-10 \%$, threshold distance $g_{v}=20 \mu \mathrm{m}$ ) in line with the known physical properties (2 $\mathrm{GPa}$ Young's modulus; $10 \mathrm{~nm}$ diameter) of IFs (Kojima et al. 1994; Gittes et al. 1993). The IFs are known to resist large deformations, whereas they are less involved in small cell deformations (Wang and Stamenovic 2000; Fudge et al. 2003). As in former CSK models (Wang and Stamenovic 2000; Sultan et al. 2004), we therefore adopted a negative prestrain, which means that the interactions will be null around the nucleus unless the medium undergoes large deformations.

The cortical actin network (or cortex) results from the Elastic Wire law $\left(K=100 \mathrm{nN}, \tau=2 \%\right.$ and $\left.g_{v}=20 \mu \mathrm{m}\right)$, between the nodes of the medium boundary, which we have called cortical nodes, as shown in Fig. 4b. The cortical nodes interact with each other or with other boundary nodes according to a Cohesive Contact law with a high cohesion value $\left(c=100 \mathrm{nN}, a=1 \times 10^{-1} \mu \mathrm{m}\right)$.

The other nodes of the medium, called the internal nodes, are the numerous species of the model forming the internal part of the medium between the nucleus and the boundary. They interact with the boundary nodes, the nucleus and the perinuclear nodes as described by the Cohesive Contact law ( $c=10 \mathrm{nN}, a=$ $5 \times 10^{-2} \mu \mathrm{m}$ ) and with each other as described by the Cohesive Contact law with a very low value of cohesion $\left(c=0.4 \mathrm{nN}, a=5 \times 10^{-2} \mu \mathrm{m}\right)$. These node species counterbalance the previously introduced tensile interactions by forming tightly compressed chains. The resulting diffuse compression network shown in Fig. $4 \mathrm{c}$ is taken to mimic the lattice of MTs, while the resulting diffuse tension network is taken to mimic the MF thin lattice.

The connectivity between nodes is not fixed and is liable to change during the numerical simulation, depending on how the node envelopes contact each other or separate. Some interactions can disappear, while others can be formed.

The distant tension network and the close tension and compression networks generated throughout the entire model are interconnected and self-balanced by adapting the connectivity between the nodes and the orientation and magnitude of the resulting forces.

\subsection{Numerical method of resolution}

The CDM model equations are solved using the LMGC90 code, which provides an open platform for modeling contact or multi-physics interaction problems. The LMGC90 code is based on Non-Smooth Contact Dynamics (NSCD), a numerical method which was developed for dealing with connecting elements in a divided medium (Moreau 1993; Jean 1999; Cambou and Jean 2001). The discrete forms of the dynamic equation
(2) are solved along with contact laws (1) and Eqs. (3) and (4) using an implicit block non-linear Gauss-Seidel algorithm (Jean 2001).

\subsection{Model implementation}

To analyze the contribution of each substructure in the CSK (i.e. actin cortex, SF network, IF network, MT network) as well as the adhesion conditions on which the overall mechanical responses of the cell (stressstrain relationship, stiffness) depend, as often observed in cells in vitro (Heidemann et al. 1999; Laurent et al. 2002; Wang et al. 2002; Chen et al. 2003; Smith et al. 2003), five configurations of the CDM model are tested. In each configuration, one of the CSK substructures is changed or removed. The overall mechanical response of the CDM model in each of these configurations is then compared with that of the model in the "complete CSK" configuration. The complete CSK configuration is obtained with all the various node species and associated interaction laws describing all the CSK features. In the "cortex-free CSK" configuration, both the Elastic Wire interaction law and the high Cohesive Contact interaction law between the cortical nodes are removed. The cortical nodes are therefore regarded here as simple internal nodes involved in low-cohesion contact interactions. The cortex-free CSK configuration could be used to analyze the role of the cortical tension. In the $T N$-free $C S K$ configuration, the Elastic Wire interaction laws between the focal adhesion nodes, the perinuclear nodes and the nucleus are removed. This configuration could simulate the cultured adherent cells drugged with cytochalasin $\mathrm{D}$, where the SF network is dismantled (Wang et al. 1993; Wendling et al. 2000). The "low adhesion CSK" configuration is equipped with only half of the focal adhesion nodes. The "IF-free CSK" configuration lacks the Elastic Wire interaction law between the perinuclear nodes and the nucleus. In the "Weak-MT CSK" configuration, the internal nodes in the divided medium interact according to the soft Cohesive Contact law with a compressive rigidity $S=100 \mathrm{nN}$. The two latter configurations can be used to analyze the contributions of IFs and MTs to cellular responses.

The CDM model is tested under controlled and quasi static conditions by mimicking biological experiments using the microplate device to analyze the overall mechanical behavior of single adherent cells in vitro (Thoumine and Ott 1997; Desprat et al. 2005; Micoulet et al.2005). The experimental microplate device imposes an overall deformation on the cell by compressing and stretching a single cell held between a rigid microplate 

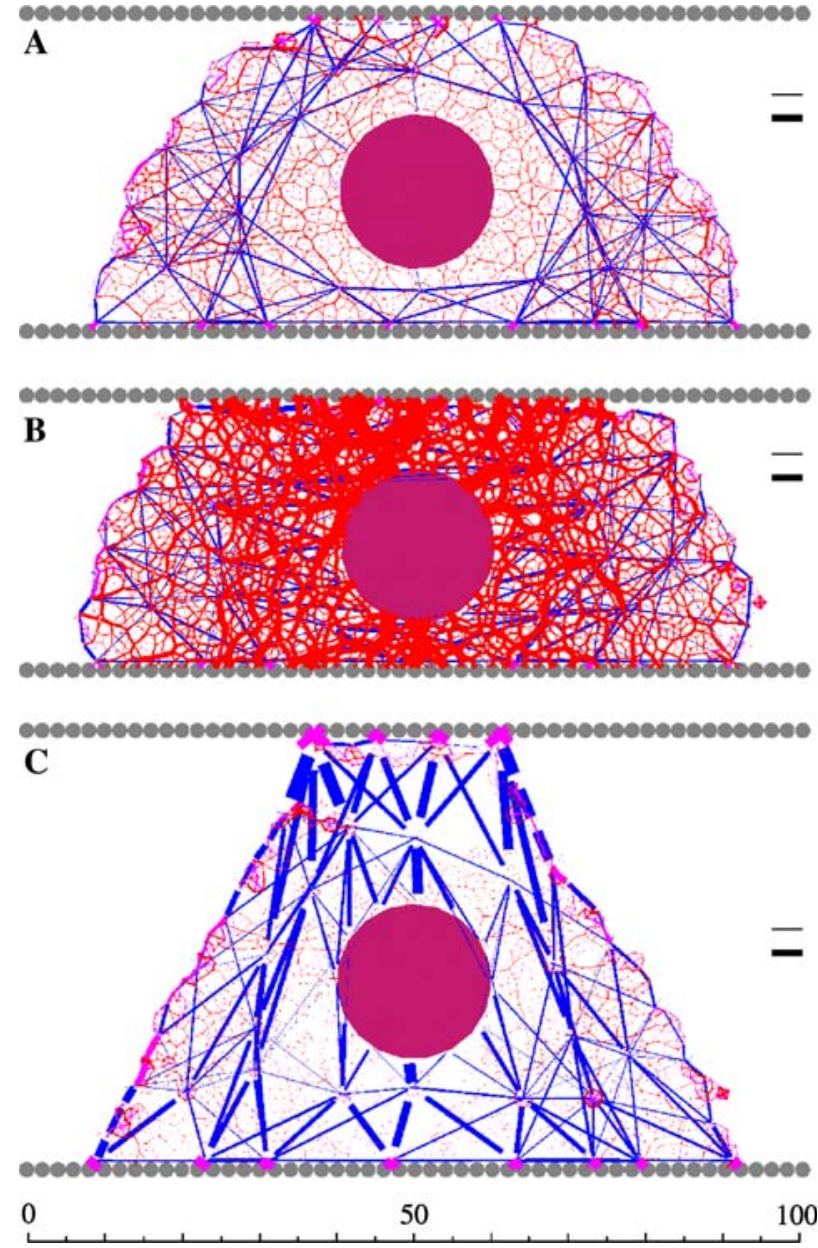

Fig. 5 Internal force distribution in the complete CSK configuration $\mathbf{a}$ in the reference state, $\mathbf{b}$ in the $14 \%$ compressed state and c in the $40 \%$ stretched state. Blue lines give the distant tension forces, red segments the close compression forces and purple ones, the close tension forces. Bars, 20 and $100 \mathrm{nN}$. Unit of length: $1 \mu \mathrm{m}$

placed parallel to a flexible microplate used as a sensor to gauge the applied force.

At the beginning of the simulation, the CDM model is held between two parallel rigid plates and interacts with them via the focal adhesion nodes, as show in Fig. 5a; this initial state is taken as the reference state in the compression/stretching tests. The lower plate is fixed, whereas the upper one is mobile and normally applies to the lower plate a compression load along an axis taken by convention to be negative and a stretching load in the opposite direction. The model is first compressed until $14 \%$ deformation is reached and then stretched until $40 \%$ deformation is reached.

During the simulation, the adhesion conditions do not change from the reference state. No new focal adhesions appear in the compression test, nor do any of the existing ones disappear in the stretching test. During the numerical compression and stretching simulations, the upper plate moves at a speed of $\pm 5 \times 10^{-2} \mathrm{~m} / \mathrm{s}$. The time step is $0.2 \mu \mathrm{s}$ and 50 iterations per step are performed on the model during the simulation. The mass of the nodes is proportional to the virtual volume enclosed by their envelope, seen in 3D as a sphere. The volumic mass is $1 \mathrm{pg} / \mathrm{\mu m}^{3}$ and the node mass ranges between 0.6 and $2.6 \mathrm{pg}$. In this simulation, the node mass is not significant. The local acceleration forces remain negligible with respect to the externally applied forces, as indicated during the computation by the very low values $\left(10^{-10}\right)$ of the dynamic/static criterion which means that the conditions simulated by the CDM model are quasi static.

To analyze the mechanical behavior of the model, an equivalent stress $\sigma$ is defined by the ratio between the external force $F$ applied to the model by the upper plate and $\Phi_{c}$, the fictive horizontal cross-sectional area of the model measured at the level of the nucleus center:

$\sigma=\frac{F}{\Phi_{c}}$

The apparent strain $\varepsilon$ in the overall model is defined as the deformation along the applied normal force axis,

$\varepsilon=\frac{H-H_{0}}{H_{0}}$

where $H$ (respectively, $H_{0}$ ) is the height of the current state of the model along the applied force axis (respectively, in the reference state).

To estimate the apparent stiffness of the CDM model at low deformation levels (near the reference state), an equivalent elasticity modulus $E$ is calculated at $2 \%$ deformation under compression and stretching conditions.

$E=\left(\frac{\Delta \sigma}{\Delta \varepsilon}\right)_{\varepsilon=2 \%}$

The distant tension forces generate internal tension and give to the model a prestress. The prestress $P$ is defined by the ratio between the sum of all these distant tension forces $T_{i}$ and the fictive cross-section $\Phi_{b}$ of the basal side of the model.

$P=\frac{\sum_{i} T_{i}}{\Phi_{b}}$

The value of the prestress varies as a function of the prestrain $\tau$ defined in the elastic law (see 7). The prestress computed in the reference state defines the initial prestress $P^{*}$. The changes in $P$ occurring during compression and stretching are then compared with $P^{*}$. 


\section{Results}

The results of the present numerical simulations carried out using the CDM model can be seen in Figs. 5-8, which give the internal force distributions in the reference state and in the greatly compressed and stretched states in the complete, cortex-free, $\mathrm{TN}$-free and low adhesion configurations of the CDM model. Figure 5 shows the complete CSK configuration with the cortical network, the large and perinuclear networks of distant tensions, as well as the diffuse tension and compression networks. It can be observed that, in comparison with the reference state (Fig. 5a), the organization and orientation of these networks differ in both the compressed (Fig. 5b) and stretched states (Fig. 5c). In the 14\% compressed complete CSK configuration shown in Fig. 5b, a highly diffuse compression network is formed throughout the entire model from the upper plate to the lower plate in the loading direction and arround the nucleus. In addition, the internal distant tensions form a strong network between the nucleus and the free boundary of the model, running perpendicular to the loading direction and preventing the widening of the medium. The highly diffuse compression network which develops during the compression loading almost disappears under stretching conditions as shown in Fig. 5c, while the distant tension network which is connected to both plates via focal adhesion points is strengthened and undergoes an overall reorganization in the stretching direction. In addition, in the complete CSK configuration of the model subjected to compression and stretching, the prestress $P$ compared to $P^{*}=276 \mathrm{~Pa}$ increases twice as much in response to compression as to stretching (45\% at $14 \%$ compressed state and $22 \%$ at $14 \%$ stretched state). During the stretching test, the free edges of the model retract toward the nucleus due to both the internal tension and the cortical tension which ensures the integrity of the CDM model (see Fig. 5c).

Removing the cortical network from the model weakens the distant tension network, as shown by the cortex-free CSK configuration of the model in Fig. 6a. In this configuration, the CDM model loses the cortical tension and some internal distant tensile forces normally exerted on the cortex. The initial prestress $P^{*}=213 \mathrm{~Pa}$ in this configuration is $22 \%$ lower than in the complete CSK configuration. During compression, the diffuse compression network becomes weaker than in the complete CSK configuration (see Fig. 6b). During stretching, the internal force network is concentrated in a region closely surrounding the nucleus, and the model shows a wide edge band which is almost mechanically inert. Without the tensile cortex, the internal distant tension network keeps the same orientations as in the
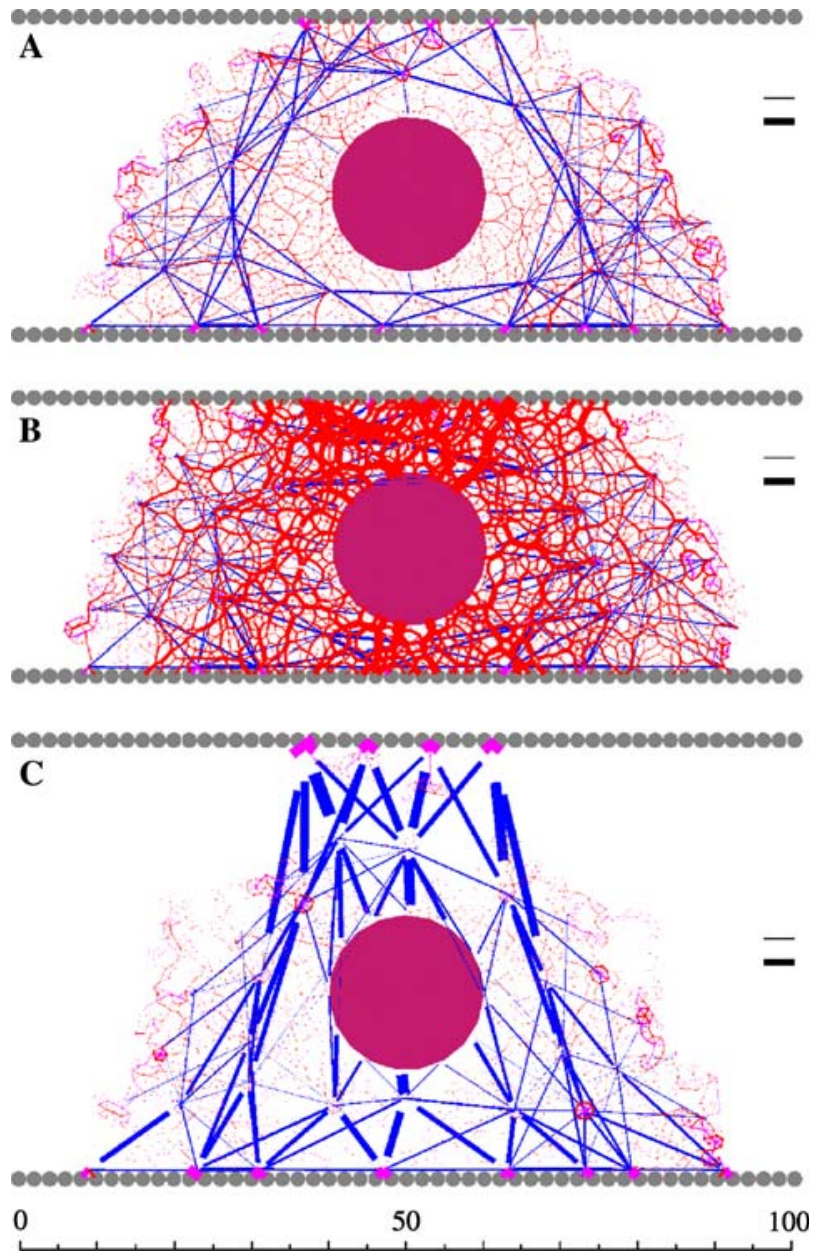

Fig. 6 Internal force distribution in the cortex-free CSK configuration $\mathbf{a}$ in the reference state, $\mathbf{b}$ in the $14 \%$ compressed state and c in the $40 \%$ stretched state. Blue lines give the distant tension forces, red segments the close compression forces and purple ones the close tension forces. Bars, 20 and $100 \mathrm{nN}$. Unit of length: $1 \mu \mathrm{m}$

complete configuration. The diffuse compression network only develops between the internal nodes of these distant tension network (see Fig. 6c).

In the TN-free CSK configuration, the cortex is the only active tensile network which creates a cortical tension and maintains the integrity of the CDM model as well as the connection between the upper and lower plates (see Fig. 7a). In this TN-free CSK configuration, the internal large and perinuclear distant tension networks are removed and the initial prestress $\left(P^{*}=\right.$ $59 \mathrm{~Pa}$ ), which is generated only by the cortex, is $79 \%$ lower than that occurring in the complete CSK configuration. During compression loading, a weak, diffuse compression network develops through the model, directed toward the nucleus (see Fig. 7b). During stretching, the nucleus appears to be totally disconnected from the medium boundary. No significant internal forces 

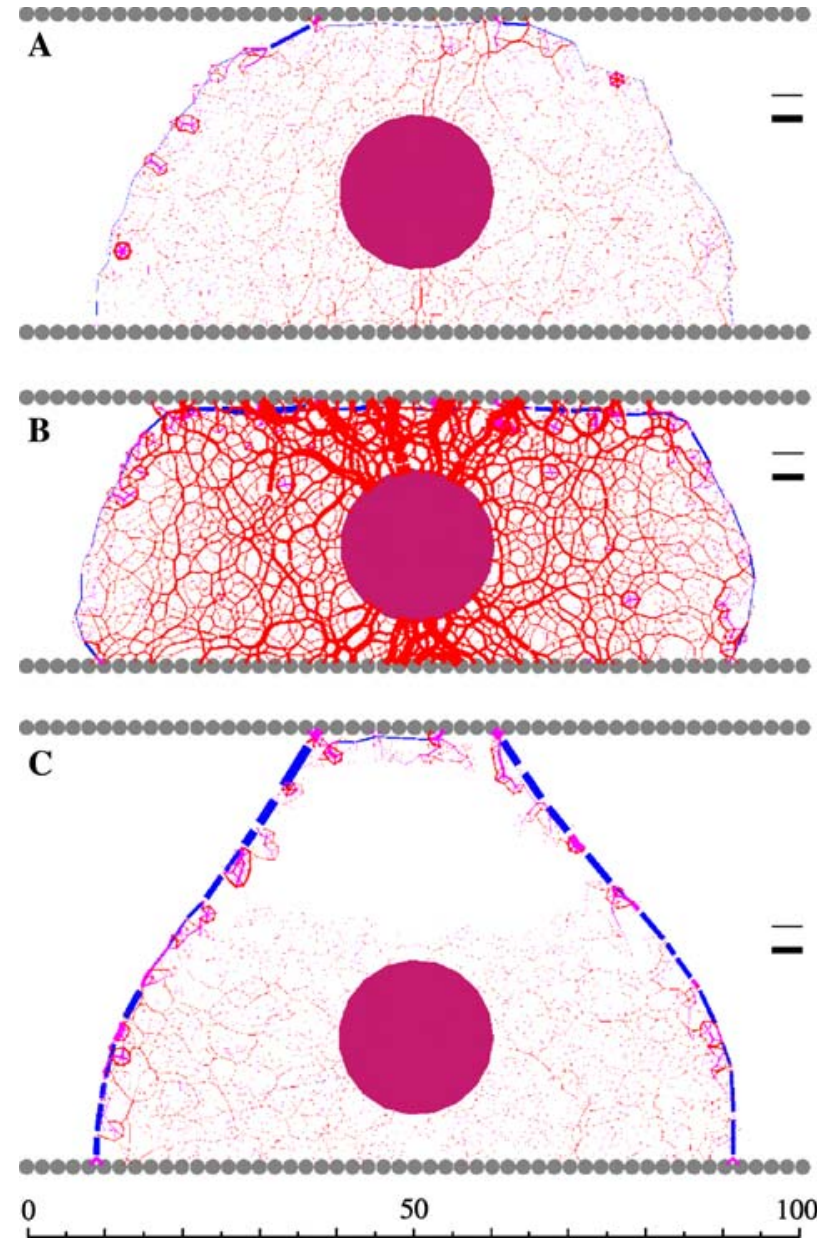

Fig. 7 Internal force distribution in the TN-free configuration a in the reference state, $\mathbf{b}$ in the $14 \%$ compressed state and $\mathbf{c}$ in the $40 \%$ stretched state. Blue lines give the distant tension forces, red segments the close compression forces and purple ones the close tension forces. Bars, 20 and $100 \mathrm{nN}$. Unit of length: $1 \mu \mathrm{m}$

develop here through the model: the diffuse compression network completely disappears and the diffuse network of tension becomes very weak (see Fig. 7c).

Reducing the number of adhesion points in the low adhesion configuration of the model, affects the distant tension distribution in the reference state and during stretching, but has no effect on the internal force distribution of the model during compression as shown in Fig. 8 as compared to Fig. 5. The initial prestress $\left(P^{*}=\right.$ $251 \mathrm{~Pa}$ ) is slightly affected and is $9 \%$ lower than in the complete CSK configuration.

The CDM model shows non-linear stress-strain relationships during compression and stretching in all six configurations (see Figs. 9, 10). During compression, the stress magnitude varies in the $[0 \mathrm{~Pa} ; 248 \mathrm{~Pa}]$ range at strain rates in the $[0,14 \%]$ range. The elasticity modulus, which is the slope of the stress-strain curves measured at $2 \%$ strain is quite similar in the low adhesion
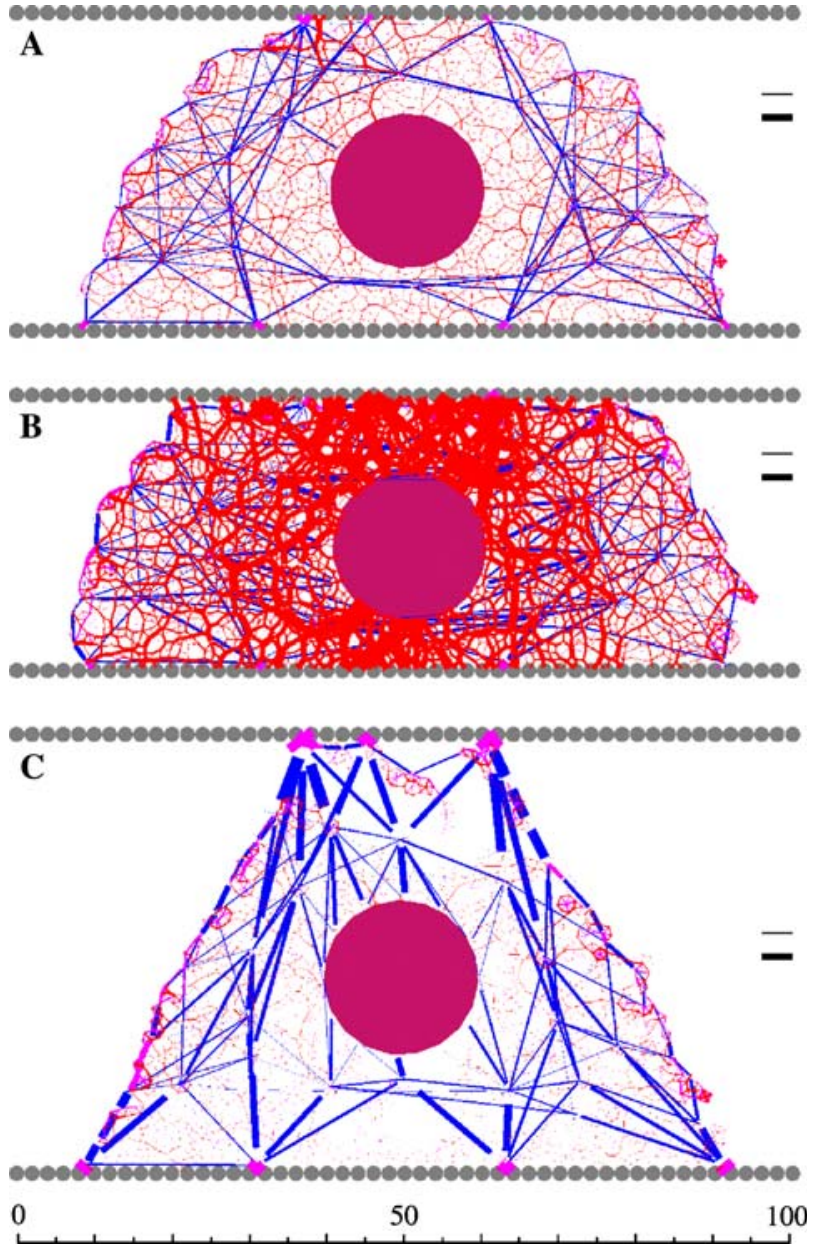

Fig. 8 Internal force distribution in the low adhesion CSK configuration $\mathbf{a}$ in the reference state, $\mathbf{b}$ in the $14 \%$ compressed state and $\mathbf{c}$ in the $40 \%$ stretched state. Blue lines give the distant tension forces, red segments the close compression forces and purple ones the close tension forces. Bars, 20 and $100 \mathrm{nN}$. Unit of length: $1 \mu \mathrm{m}$

$\operatorname{CSK}(E=1,297 \mathrm{~Pa})$, the complete $\operatorname{CSK}(E=1,287 \mathrm{~Pa})$ and the cortex-free CSK $(E=1,215 \mathrm{~Pa})$ configurations, while a significantly low level of stiffness is observed in the $\mathrm{TN}$-free $\mathrm{CSK}$ configuration $(E=717 \mathrm{~Pa})$, where a significant decrease in the stiffness occurs in the early stages of deformation (see Fig. 9).

In stretching tests starting with the previous 14\% compressed state of the medium and reaching a $40 \%$ stretched state, the stress magnitude varies in the $[-248 \mathrm{~Pa} ; 172 \mathrm{~Pa}]$ range in the four configurations of the model. The stress-strain curves are non-linear and can be described by a double parabolic curve showing a change of curvature. The slopes of the curves decrease until $\varepsilon=-7 \%$, and then increase during model stretching, reflecting the strain-hardening behavior of the model. In addition, the slopes of the curves obtained during the first stage of stretching are lower than during 


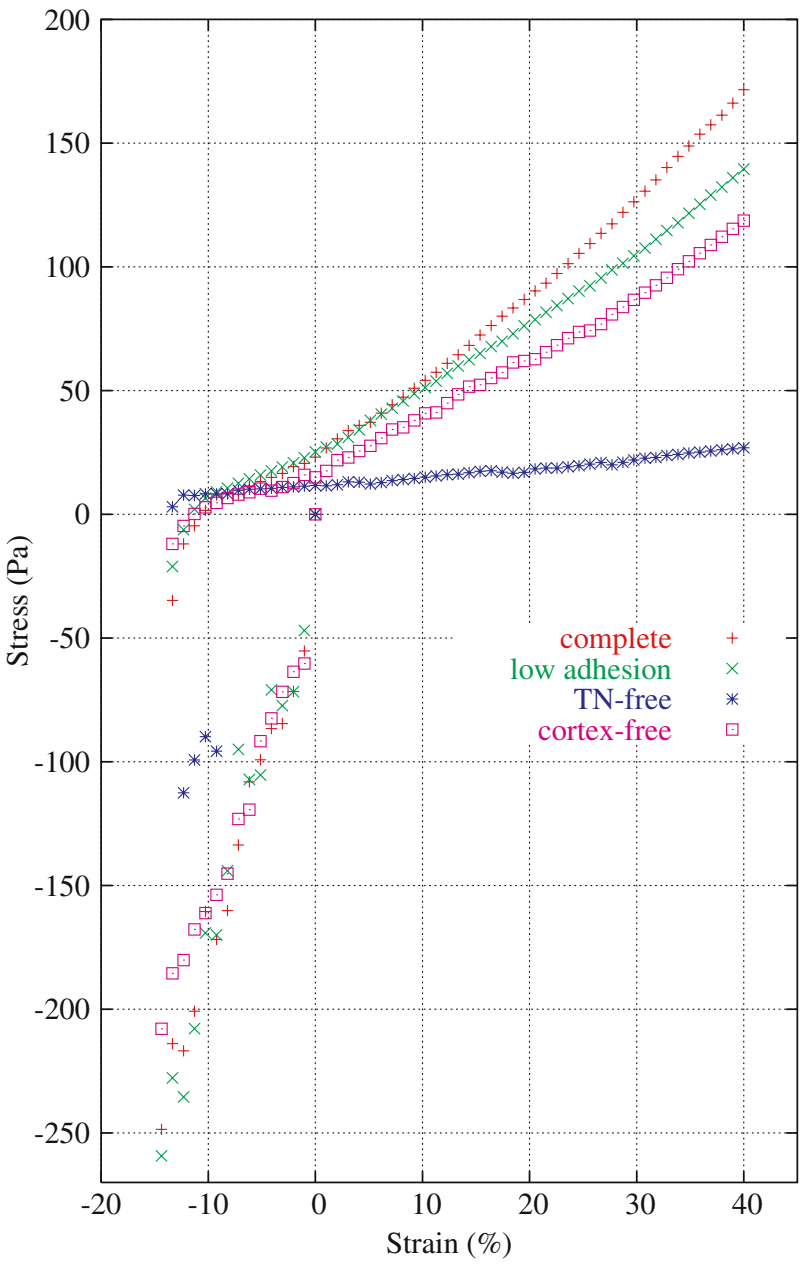

Fig. 9 Stress-strain relationships given by the complete, cortexfree, TN-free and low adhesion CSK configurations of the CDM model under uniaxial compression and stretching, successively. Stress and strain are both taken to be positive in the stretching direction

compression, which means that the CDM model is more flexible during small stretching deformation than during compression. The complete CSK configuration appears to be stiffer $(E=382 \mathrm{~Pa})$ than the low adhesion CSK $(E=326 \mathrm{~Pa})$, the cortex-free $\operatorname{CSK}(E=286 \mathrm{~Pa})$ and the TN-free CSK $(E=86 \mathrm{~Pa})$. This configuration also shows the greatest strain-hardening behavior, as indicated by the sharp increase in the slope of the curve (see Fig. 9); whereas the TN-free CSK configuration, which is the less stiff configuration, shows a significantly low stiffening behavior during stretching.

It is worth noting that during stretching, when the model recovers zero strain, the resulting stress applied is non-null (i.e. the [10-25 Pa] range in all four configurations). The current state therefore differs from the initial reference state of the model (i.e., $\varepsilon=\sigma=0$ ). This state is reached quickly in both $\mathrm{TN}$-free CSK and cortex-free

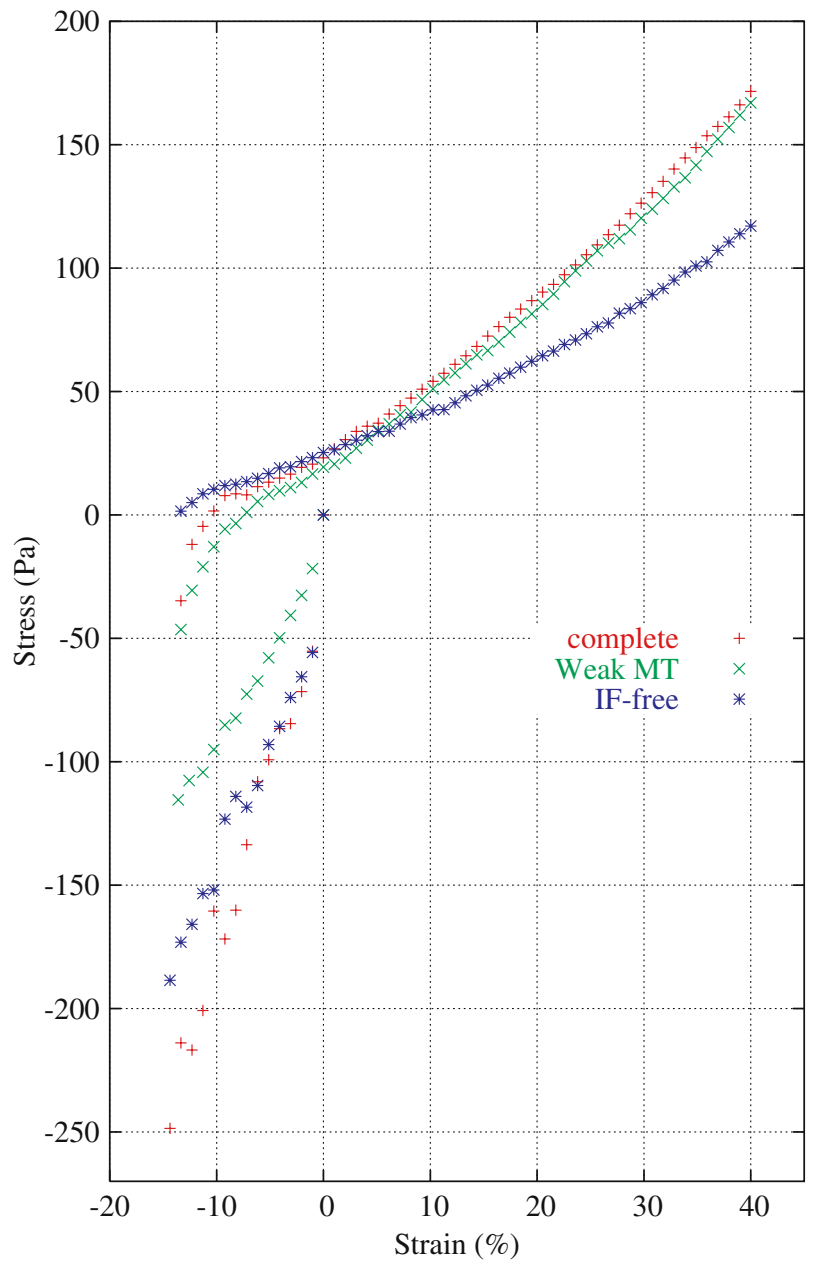

Fig. 10 Stress-strain relationships given by the complete, Weak MT and IF-free CSK configurations of the CDM model under uniaxial compression and stretching, successively. Stress and strain are both taken to be positive in the stretching direction

CSK configurations, because in these configurations the model is less prestressed and more flexible than in the complete CSK and low adhesion CSK configurations. In addition, a hysteresis occurs in the strain-stress curve between compression and stretching loading conditions in all six configurations of the model (see also Fig. 10).

The stress-strain relationships given by the model in the Weak-MT CSK and IF-free CSK configurations are compared with the complete CSK configuration in Fig. 10. The stress-strain curve given by the model in the Weak-MT CSK configuration shows a lower slope $(E=777 \mathrm{~Pa})$ during compression than in the complete CSK configuration $(E=1,287 \mathrm{~Pa})$ but the results are similar during large stretching deformations. Besides the low stiffness observed during compression, the model in the Weak-MT CSK configuration slowly reaches the zero stress state and the hysteresis therefore decreases. 
By contrast, in the IF-free CSK configuration, the slope of the curve decreases significantly at large stretching deformations, which means that the strain-hardening is less pronounced than in the complete CSK configuration of the model. During compression, removing the IF network has little effect on the stress-strain curve. The zero stress state after compression is reached faster in this configuration than in the complete and the weak MT configurations of the model.

\section{Discussion}

The numerical results obtained with the present CDM model suggest that during cell deformation, the CSK is rearranged depending on the internal tension/ compression distribution which results from interactions occurring and disappearing between nodes within the medium. Interactions of two kinds are defined in the model, which are described by a contact law between rigid envelopes of neighboring nodes and an elastic law between remote nodes. The contact law results in diffuse networks of compression and tension, and the elastic law results in large and perinuclear distant tensile networks. Both interaction laws defined between nodes located on the boundary of the medium results in the so-called cortical network. These force networks are balanced to stabilize the model during deformation.

The mechanical behavior of the CDM model and the internal structural changes occurring in this model under quasi static loading conditions suggest several analogies with the mechanical responses of adherent cells. In fact, assuming that the SF and IF tensile networks which extend through the whole cell, are simulated in the CDM model by the deep distant tension network (TN), the results suggest the occurrence of SF and IF alignment and a thickening in the stretching direction, as well as an increase in the internal prestress levels which is closely related to the density of the SFs (Smith et al. 2003; Meazzini et al. 1998). In addition, these two CSK tensile networks are distinguished. The perinuclear distant tension network reflects the behavior of the IFs mostly concentrated in the perinuclear region and the large distant tension network reflects the behavior of the SFs which are mostly concentrated in the basal region and extend through the whole cell, reaching the apical side (Coulombe et al. 2000; Katoh et al. 1998).

The IFs are not specifically identified in the model in the peripherical region. However, the model may simulate the IF network extensions through the cell and to the membrane, and some forces in the large distant tension network mainly carried by the SFs may also be carried by the IFs. In addition, the diffuse compression network which is formed by chains of close compression forces in the direction of the applied force, is assumed in adherent cells to correspond to the lattice of microtubules (MTs), which have been reported to resist external compression (Janson et al. 2003; Kurachi et al. 1995; Heidemann et al. 1999) and to disappear during stretching (Ingber 2003; Meazzini et al. 1998). It is worth noting that in our model, the nucleus is assumed to be a rigid solid, as proposed by Heidemann and Wirtz (2004), and the CDM diffuse tension network resulting from local tension interactions is assumed to correspond to the MF thin network (Katoh et al. 1998; Satcher and Dewey 1996).

\subsection{Dynamic organization of the CSK structure}

Under externally applied forces, the diffuse compression network is extremely unstable, and shows fast changes in connectivity between the nodes involved in chains of close compressions. Let us recall here that in the CDM model, the diffuse compression network involves several close contacts between node envelopes. During the deformation of the model, these close compression forces can either disappear, change their direction, decrease or increase in magnitude to locally balance the close and distant tension forces as well as the externally applied forces. By contrast, the distant tension network is exerted only on remote nodes, and is far less affected by changes in connectivity unless new focal adhesion points are activated. This network rearranges itself only via changes in the magnitude and orientation of the forces. For instance, when the model is submitted to compression and extension loadings in the $[-14 \%$; $40 \%$ ] range of overall deformation, few distant tensile forces occur or disappear and the connectivity remains practically unchanged and stable. The distance $(10 \mu \mathrm{m})$ between these remote nodes is one order of magnitude greater than the distance $(1 \mu \mathrm{m})$ between nodes in close contact. Therefore, the diffuse compression network, which is carried by closely contacting nodes, is more likely to be affected by deformations than the distant tension network, which is based on interactions between remote nodes.

These results are consistent with the unstable behavior of the MT network, as compared with the stable behavior of the SF network, previously described by Kaverina et al. (1998) and Katoh et al. (1998). The connectivity of the diffuse compression network is more variable than that of the SF network because the characteristic length of $1 \mu \mathrm{m}$ is smaller than the $10 \mu \mathrm{m}$ characteristic length of the SF network. Contrary to the MT network, the stress fibers may undergo large 
deformations induced by flexible substrate retraction $(20 \%$, Sato et al. 2005$)$ or by cell stretching between microplates (150\%, Micoulet et al. 2005) without dismantling. These mechanical responses may be attributable to MT polymerization/depolymerization processes which occur within $1 \mathrm{~min}$ of deformation (i.e., the polymerization speed is approximately $1 \mu \mathrm{m} / \mathrm{min}$ ) while a longer time is required for the $10 \mu \mathrm{m}$ stress fibers to form (Janson et al. 2003; Kaverina et al. 1998). Therefore the CDM model shows the changes in connectivity and overall distribution of the internal forces that occur during the short mechanical responses of adherent cells. The polymerization processes which occur during longterm adaptation of the cell are not taken into account in the present model.

The laws governing the interactions between nodes do not take the viscosity into account but the changes in connectivity occurring during the overall deformation of the medium involve irreversible rearrangements, which give rise to mechanical dissipation in the CDM model. This dissipation can be quantified and shows up in the stress-strain curves in the form of a region of hysteresis. The dissipative behavior of the CDM model therefore differs from the viscous behavior of living cells observed under dynamic conditions using microrheological methods (Kole et al. 2004, 2005). However, in view of the hysteresis observed in vitro using atomic force microscopy and the cell-poking apparatus (Petersen et al. 1982; Collinsworth et al. 2002), the CDM model suggests that local cytoskeletal rearrangements may be involved in the dissipative mechanical behavior of the cell in addition to the viscous CSK filaments and cytoplasm.

The changes of connectivity occurring in the CDM model might also explain the local changes in the pattern of CSK organization due to local disruptions/ connections occurring within the actin filament and microtubule networks. However, the present model is not able at the present state to describe the cross-linking between filaments on the molecular scale. Studies on what it is called the progressive refinement or fragmentation of the divided medium are still in progress. Thus the present model could be improved by refining the interactions between nodes, which would make it possible to analyze the cross-linking proteins known to enhance the stiffness of cells (Tseng et al. 2004; Kaverina et al. 1998).

\subsection{Adaptation of the CSK to the mechanical environment}

The present CDM model also shows how the CSK adapts its shape and structural distribution to the mechanical microenvironment so that a balance is reached between the CSK filamentous networks and the extracellular matrix $(\mathrm{ECM})$. The CDM model is significantly strengthened in response to the external stress applied during mechanical loading. For instance, in response to $40 \%$ stretching, the prestress $P$ increases twofold in comparison with $P^{*}$. To withstand the increasing internal tension, the adhesions are then reinforced and the tensions between focal adhesion nodes and the plates increase by $250 \%$ on average. The CDM model therefore shows that the focal adhesions become stronger as the prestress increases, as previously observed in cells in vitro (Wakatsuki et al. 2000; Bershadsky et al. 2003). When the number of focal adhesion points decreases, the overall mechanical response of the model does not change significantly (see Fig. 9). The diffuse compression network is slightly reinforced (see Fig. 8b compared to Fig. 5b during compression). During stretching, the low adhesion configuration of the model shows a less densely structured SF network, and the strengthening of the focal adhesion points and the cortical network balances the externally applied forces. The tensile force per focal adhesion point (FAP) increases on the basal side with the extension of the model and is $36 \%$ higher in the $10 \%$ stretched state (and 10\% higher in the reference state) in the low adhesion configuration than in the complete CSK configuration. It turns out that a decrease in the number of focal adhesion points does not affect the prestress, but increases the force at the adhesion sites, as found to occur in adherent myocyte cells (Griffin et al. 2004) and fibroblasts upon measuring the forces exerted on individual focal adhesions (Balaban et al. 2001; Tan et al. 2003). The present results also suggest that a minimum number of focal adhesion points are necessary to keep the cell stably anchored to the substrate. The CDM model could therefore help to determine this minimum at a given level of prestress and a given state of deformation.

These results are also comparable to those obtained in a previous in vitro study on adherent cells (Wendling et al.2000). In this study, we observed that confluent and non-confluent adherent cells, which have very differently organized SF networks, also showed similar stiffness. In non-confluent cells, stress fibers appear to be mainly located in the basal region of the cell and involve a large number of focal adhesion points bound to the ECM while in confluent cells, the stress fibers are mainly located in the cortex of the cell, which interacts with the neighboring cells. The CDM model shows that the prestress is generated by both the cortex and the internal SF network, depending on the state of cells induced by the microenvironment, i.e., depending on whether it is a spread cell adhering to the ECM, a spread cell in confluence or a round cell in suspension. 


\subsection{Mechanical response of the CSK structure}

The 2D CDM model was tested under compression and stretching loading with a constant rate of deformation, and the numerical results obtained in terms of the stress-strain relationships and the elasticity modulus are compared with those measured in microplate experiments performed on isolated cells in vitro (Thoumine and Ott 1997; Desprat et al. 2005; Micoulet et al. 2005).

The resulting stresses applied to the model in the complete CSK configuration (248 Pa at $14 \%$ of compression; 107 and $172 \mathrm{~Pa}$ at 25 and $40 \%$ of stretching) are of a similar order of magnitude to those of experimental data [e.g., $\sigma=500 \mathrm{~Pa}$ at $25 \%$ stretching (Micoulet et al. 2005), $\sigma=66 \mathrm{~Pa}$ at 23\% stretching (Desprat et al. 2005), $\sigma=200-600 \mathrm{~Pa}$ at $25 \%$ compression or stretching (Thoumine and Ott 1997)].

The complete CSK configuration of the CDM model shows an elasticity modulus $E=1,287 \mathrm{~Pa}$ under compression and $E=382 \mathrm{~Pa}$ under stretching. These values are similar to those $(E=660 \mathrm{~Pa})$ measured by Desprat et al. (2005) in a study on cell creep responses using a microplate device and those ( $E \simeq 710 \mathrm{~Pa})$ measured by Alcaraz et al. (2003) using an atomic force microscopy device, as well as being in the $[300 ; 3,000 \mathrm{~Pa}]$ range measured by Fabry et al. (2003) using a magnetocytometry device on several types of cells.

The non-linearity of the stress-strain curves reflects the stiffening behavior of the CDM model, i.e., the stiffness increases with the applied strain, which was also observed experimentally in cultured cells by several authors (Wang et al. 1993; Wang and Ingber 1994; Alcaraz et al. 2003; Laurent et al. 2002) and in theoretical CSK models (Stamenovic et al. 1996; Coughlin and Stamenović 1997, 1998; Wendling et al. 1999; Volokh et al. 2000). Although Thoumine and Ott (1997), Desprat et al. (2005) and Micoulet et al. (2005) have observed linear mechanical responses in single cells tested in vitro with a similar microplate device in wide stretching ranges $(0<\varepsilon<400 \%)$, Desprat et al. (2005) have also reported that non-linearity occurs at low strain levels $(\varepsilon<1 \%)$. This non-linearity, which also occurs in the CDM model at the beginning of stretching tests, might be taken to be a structural response involving the early reorganization of the SFs and IFs in the stretching direction, whereas linear stress-strain relationships at large deformation rates may result from the intrinsic deformation of these filaments.

It is to notice that in the CDM model the linear interaction laws which represent an approximation of the mechanical behavior of the CSK filaments may lead to an underestimation of the overall stiffness of the cell at large deformations.

\subsection{Contribution of the CSK substructures} to the overall mechanical behavior of adherent cells

The changes in the overall mechanical responses of cells subjected to external forces partly depend on the distribution of the forces within the structural components of the cell and the way these cellular components act together in response to externally applied forces. The CDM model helps to establish the mechanical roles of the various CSK substructures and suggests in addition that close relationships between the nucleus, the actin-based cortex, the focal adhesion points and the CSK filamentous networks are required to give integrity to the whole cell (Laurent et al. 2002; Ingber 2003; Heidemann and Wirtz 2004; Wang et al. 2001).

The results obtained here with the CDM model show that the tensile network (by analogy with the SF network) plays a fundamental role in generating the overall prestress and in controlling the stiffness of the cell. For instance, removing this tensile network from the CDM model decreased $P^{*}$ by $79 \%$ and $E$ by $44 \%$ in compression and by $77 \%$ in stretching tests. The linear $\left(E-P^{*}\right)$ relationship obtained with the CDM model reflects a prestress-induced stiffening behavior as shown in Fig. 11a. This prestress dependence of the overall stiffness is consistent with the results of previous studies on adherent cells (Wang et al. 1993, 2001, 2002; Wang and Ingber 1994; Hubmayr et al. 1996; Pourati et al. 1998; Thoumine and Ott 1997; Wendling et al. 2000; Laurent et al. 2002) as well as those of theoretical studies on structural models (Stamenovic et al. 1996; Stamenovic and Coughlin 1999; Canadas et al. 2002; Wendling et al. 2003; Coughlin and Stamenović 2003; McGarry and Prendergast 2004).

During extension tests, the integrity of the CDM model is lost when the tensile network is removed (i.e., $\mathrm{TN}$-free CSK configuration): the nucleus is no longer connected to the cortex under these conditions. The cortex alone gives the CDM model its ability to resist the overall deformation. This result may explain the different levels of overall stiffness measured in both adherent cells and non-adherent cells. In fact, the magnetocytometry measurements performed by Laurent et al. have shown that the stiffness was greater in adherent cells than in cells in suspension, and a $3 \mathrm{D}$ reconstruction of these cells showed the presence of (1) a strong CSK structure with a dense SF network anchored to the ECM via the focal adhesion points in the adherent cell, and (2) a 2D submembranous actin lattice in the cell in suspension (Laurent et al. 2003).

During compression tests, the integrity of the CDM model is reduced but not lost and externally applied stresses are transmitted from the cortex to the nucleus 
through both the diffuse tension network simulating the thin MF network and the diffuse compression network simulating the MT network. In addition, the model shows a loss of integrity when the cortex is removed. Although the SF network is anchored to the ECM on the basal side via the focal adhesion points, the model suggests that this tensile network needs connections with the cortical tension network to be able to distribute internal tension throughout the cell and to anchor efficiently to precursors of adhesion points on the apical side. When these adhesion sites are not connected to the cortex, the SFs and IFs cannot link them (Marek et al. 1982; Katoh et al. 1998). Although this cortex-free configuration is not directly observed in adherent cells in vitro, the model shows how important the surface or membrane tension is for the most efficient cytoskeleton reorganization that occurs through the whole cell during deformations.

The IF network simulated by the perinuclear distant tension network in the CDM model seems to also play a crucial role during large stretching deformations (see Fig.10) as well as contributing to the stability of the nucleus (Wang and Stamenovic 2000; Maniotis et al. 1997). These IFs are known to act as molecular guy wires to stiffen the nucleus mechanically and anchor it in place, whereas MTs hold the IF lattice open and stabilize the nucleus against lateral compression forces.

The MT network in the CDM model is weak during stretching loading, but seems to play a decisive role during compression loading, as indicated by the high compression forces (see Figs. 5b, c) and the high compression stiffness $(E=1,287 \mathrm{~Pa})$ recorded here in comparison with those observed under stretching loads $(E=382 \mathrm{~Pa})$.

To analyze the contribution of the MTs to the overall mechanical response, the CDM model was then tested by varying the local compressive rigidity $S$ defined in the Soft Cohesive Contact law (see Fig. 6). In the stretching tests, the local compressive rigidity $S$ was found to draw no effect on the overall stiffness $E$. However, under compression loads $E$ increases non-linearly with $S$, tending toward a maximum value corresponding to the stiffness of the model in the complete CSK configuration (see Fig. 11b). This high value of the compression stiffness is due to the Cohesive Contact law in which impenetrability conditions are assumed to exist between the node envelopes, and which can be considered as a Soft Cohesive Contact law with an infinite value of $S$. The Cohesive Contact law seems to overestimate the mechanical behavior of MTs and the Soft Cohesive Contact law is therefore more appropriate for describing the labile MT network present in adherent cells (Heidemann et al.
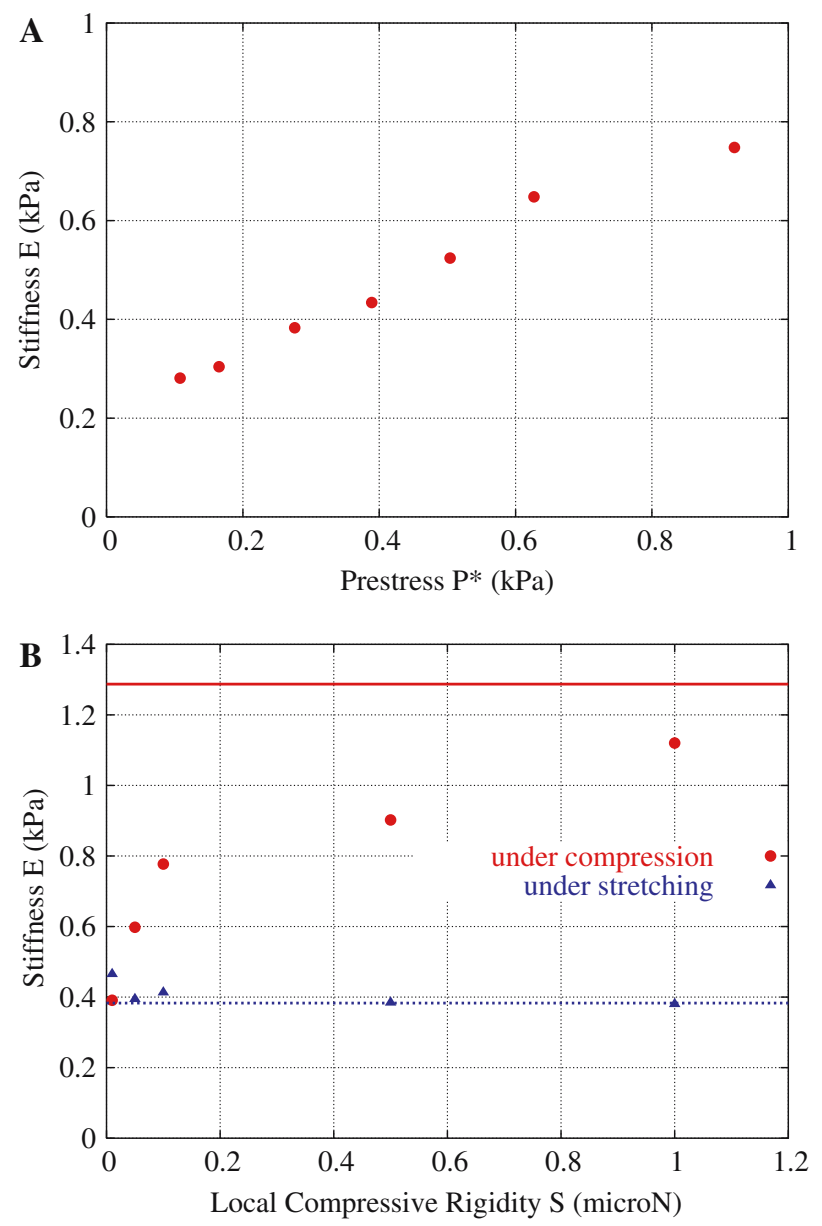

Fig. 11 a Relationship between the overall stiffness $(E)$ and the initial prestress $\left(P^{*}\right)$ given by the CDM model during stretching. $E$ was calculated at $2 \%$ strain. b Relationship between the overall stiffness $(E)$ and the local compressive rigidity $(S)$ given by the $\mathrm{CDM}$ model during compression and stretching. $E$ was calculated at $2 \%$ compression and stretching. The upper and lower lines give the values of $E$ obtained with the complete-CSK configuration of the CDM model at $2 \%$ compression and $2 \%$ of stretching, respectively

1999; Kaverina et al. 1998). However, the value of $S$ has to be identified according to biological measurements.

To summarize, these results suggest that the overall integrity of the model is reduced but not totally lost when one of the CDM model substructures (cortex, SF network, IF network, diffuse networks of MFs or MTs) or the focal adhesion points disappear during deformation. These numerical results, which are consistent with the biological findings available, show that (1) the integrity of the cell depends closely on these interconnected CSK substructures, and that (2) the connections between each of the cell components (cortex, SF network, nucleus and focal adhesion points) are crucial to the internal force transmission, the overall stiffness and the integrity of the cell (Zhen et al. 2002; Wang et al. 2002; Maniotis et al. 1997; Heidemann and Wirtz 2004). 
4.5 Comparisons with tensegrity and percolation models

The present 2D CDM model based on divided medium theory is the first attempt to analyze the mechanical behavior of living cells in terms of the dynamic reorganization of the cytoskeleton. This novel approach focuses on both the local and global structural rearrangement of the CSK occurring under externally applied stress, and the divided medium theory applied here can be said to be complementary to the tensegrity and percolation theories. In the tensegrity approach, the cytoskeleton is regarded as a force bearing structure composed of discontinuous rigid bars (by analogy with the MTs) compressed by continuous prestrained elastic (or viscoelastic) cables (by analogy with the stress fibers). The changes in the overall shape of the structure result from the equilibrium between the internal (tension and compression) forces and the external forces transmitted by the ECM. The tensegrity models developed so far have made it possible (1) to explain how local stresses trigger coordinated changes in cells and (2) to predict the material properties and architectural features of living cells, independently of the changes occurring in the CSK connections (Stamenovic et al. 2002; Wang et al. 2002; Wendling et al. 2003; Volokh et al. 2000). In the present study, we tried to overcome the limitations of classical tensegrity models, such as the small number of elements (bars and cables) involved and the fixed connectivity between these elements. Our model provides an alternative means of explaining how the organization of the filamentous CSK networks may change in response to global external loading and how different types of CSK filaments may contribute to the overall mechanical behavior of the cell. The present results show a similar overall pattern of static mechanical behavior to that observed using classical tensegrity models, i.e., non-linear stress-strain relationships, presstress-stiffness dependence, loading (compression and stretching) and the dependence of the attachment conditions on the stress-strain relationships (Stamenovic and Coughlin 1999; Volokh et al. 2000; Wendling et al. 2003; Sultan et al. 2004).

The percolation approach deals with a continuous phase transition which takes place at a critical filament concentration in the macromolecular system. Below this concentration, a phase consisting of small noninterconnected clusters occurs within the matrix. Above the critical concentration, these isolated clusters become interconnected and form a finite filamentous system (Forgacs et al. 2004). In this approach, the number of fibers in the system is finite and the connectivity depends on their concentration which can vary, whereas in the present model, the concentration (the number of nodes) remains constant and the connectivity depends on how the internal nodes interact. In addition, the filamentous network in the CDM model results from the distribution of the internal tension and compression forces and enters a prestressed state before undergoing deformation, which is not taken into account in the percolation approach. The latter approach provides a useful means of analyzing the intracellular signal transduction from the cell surface through the highly interconnected filamentous CSK to specific targets, and describing how the mechanical behavior of filamentous networks may change in response to changes in CSK polymerization or cross-linking.

The present CDM model yields a description of the CSK rearrangements from a purely mechanical point of view. In fact, the mode of CSK reorganization suggested in the model is based on the occurrence and disappearance of interactions between nodes, but it is not yet clear whether the biochemical processes of CSK filament polymerization and rearrangement occurring during the deformation of the cell can really be approached in terms of the variable mechanical connectivity described by the CDM model.

One possible means of testing the validity of the CDM model would consist in simulating different types of micromanipulation (i.e., magnetocytometry, the optical tweezers device, atomic force micrsoscopy, micropipette manipulation, substrate stretching) and fitting the data describing the overall mechanical behavior of living cells (strain, stress, stiffness and prestress) to the intrinsic parameters of the model. Another possible approach would consist in simulating the real-time CSK rearrangement observed during the deformation of cells in vitro under controlled mechanical conditions. This $2 \mathrm{D}$ CDM model, which has several limitations, could quite easily be improved by (1) using the same formulation to develop a 3D model regarding the spatial distribution of the CSK, (2) taking a non-rigid nucleus to analyze the deformation of the nucleus when the cell is subjected to externally applied forces and (3) introducing viscous interaction laws to analyze the viscoelastic behavior of the cell under dynamic loading conditions.

\section{Conclusion}

The first results obtained so far using the present 2D $\mathrm{CDM}$ model show that the divided medium theory is a useful tool for understanding CSK mechanics. The key features of the CDM model include the dynamic patterns of connectivity occuring in tension- and compression- balanced networks and the heterogeneity 
of the CSK structure. This model provides a new means of describing the dynamic reorganization of the prestressed CSK which occurs in response to changes in the mechanical environment of cells. In addition, this model can be used to specify the contributions of the various CSK substructures to cell stability.

The design of the CDM model is perfectible. More complex laws could be introduced so as to take into account the viscoelastic behavior of filaments. The constitution of the various CSK subcompartments (e.g., density and distribution of SFs and IFs, number of focal adhesion points, local compressive rigidity of the medium) could be also defined more clearly. Thanks to some features such as the so-called progressive refinement or fragmentation of the divided medium, which are still in progress, the CDM model would certainly yield new insights into the molecular mechanisms of cross-linking between the CSK filaments. The modes of CSK reorganization proposed in the CDM model and based on activation or deactivation of interactions between nodes, still require to be confirmed in further studies and to be compared with biochemical models for polymerization/depolymerization and interconnections of the CSK filaments, involved in the CSK rearrangement observed during cell deformation.

All in all, the CDM model opens new perspectives for carrying out mechanical investigations on cells. In its future versions, it may throw light on mechanical properties of cells such as the difference between local and overall behavioral scales and it may also help to elucidate more general cell processes. For instance, cell migration, which is a central process in morphogenesis, immune responses and lesion repair, could be analyzed mechanically using the CDM model to describe the dynamics of focal adhesion points (strengthening at leading edge and loosening at the tail) and the reorganization of the CSK filaments, which anchor on them. The CDM model could also be used to identify rolling or crawling behavior occuring during cell migration.

Acknowledgements We thank Patrick Cañadas (Laboratory of Mechanics and Civil Engineering, University of Montpellier) and Laurent Limozin (Laboratory of Immunology, University of Mediterranean) for many helpful discussions. This work was partly supported by grants from the "Centre National de la Recherche Scientifique" (Sciences pour l'Ingénieur) and from "La Région Provence Alpes Cote d'Azur".

\section{References}

Alcaraz J, Buscemi L, Grabulosa M, Trepat X, Fabry B, Farré R, Navajas D (2003) Microrheology of human lung epithelial cells measured by atomic force microscopy. Biophys J 84(3):20712079
Balaban NQ, Schwarz US, Riveline D, Goichberg P, Tzur G, Sabanay I, Mahalu D, Safran S, Bershadsky A, Addadi L, Geiger B (2001) Force and focal adhesion assembly: a close relationship studied using elastic micropatterned substrates. Nat Cell Biol 3(5):466-472

Bershadsky AD, Balaban NQ, Geiger B (2003) Adhesiondependent cell mechanosensitivity. Annu Rev Cell Dev Biol 19:677-695

Cambou J, Jean M (2001) Micromécanique des matériaux granulaires. Hermes Sciences, Paris

Canadas P, Laurent VM, Oddou C, Isabey D, Wendling S (2002) A cellular tensegrity model to analyse the structural viscoelasticity of the cytoskeleton. J Theor Biol 218(2):155-173

Canadas P, Wendling-Mansuy S, Isabey D (2006) Frequency response of a viscoelastic tensegrity model: structural rearrangement contribution to cell dynamics. J Biomech Eng 128(4):487-495

Chen CS, Alonso JL, Ostuni E, Whitesides GM, Ingber DE (2003) Cell shape provides global control of focal adhesion assembly. Biochem Biophys Res Commun 307(2):355-361

Chicurel ME, Chen CS, Ingber DE (1998) Cellular control lies in the balance of forces. Curr Opin Cell Biol 10(2):232-239

Collinsworth AM, Zhang S, Kraus WE, Truskey GA (2002) Apparent elastic modulus and hysteresis of skeletal muscle cells throughout differentiation. Am J Physiol Cell Physiol 283(4):C1219-C1227

Coughlin MF, Stamenović D (1997) A tensegrity structure with buckling compression elements: applications to cell mechanics. J Appl Mech 64:480-486

Coughlin MF, Stamenović D (1998) A tensegrity model of the cytoskeleton in spread and round cells J Biomech Eng 120(6):770 777

Coughlin MF, Stamenović D (2003) A prestressed cable network model of the adherent cell cytoskeleton. Biophys J 84(2 Pt 1):1328-1336

Coulombe PA, Bousquet O, Ma L, Yamada S, Wirtz D (2000) The 'ins' and 'outs' of intermediate filament organization. Trends Cell Biol 10(10):420-428

Davies PF, Robotewskyj A, Griem ML (1994) Quantitative studies of endothelial cell adhesion. Directional remodeling of focal adhesion sites in response to flow forces. J Clin Invest 93(5):2031-2038

Deguchi S, Ohashi T, Sato M (2005) Tensile properties of single stress fibers isolated from cultured vascular smooth muscle cells. J Biomech 38:1751-1759

Desprat N, Richert A, Simeon J, Asnacios A (2005) Creep function of a single living cell. Biophys J 88(3):2224-2233

Fabry B, Maksym GN, Butler JP, Glogauer M, Navajas D, Taback NA, Millet EJ, Fredberg JJ (2003) Time scale and other invariants of integrative mechanical behavior in living cells. Phys Rev E 68:041914-32

Forgacs G, Yook SH, Janmey PA, Jeong H, Burd CG (2004) Role of the cytoskeleton in signaling networks. J Cell Sci $117(\mathrm{Pt}$ 13):2769-2775

Fudge DS, Gardner KH, Forsyth VT, Riekel C, Gosline JM (2003) The mechanical properties of hydrated intermediate filaments: insights from hagfish slime threads. Biophys J 85(3):20152027

Gittes F, Mickey B, Nettleton J, Howard J (1993) Flexural rigidity of microtubules and actin filaments measured from thermal fluctuations in shape. J Cell Biol 120(4):923934

Griffin MA, Engler AJ, Barber TA, Healy KE, Sweeney HL, Discher DE (2004) Patterning, prestress, peeling dynamics of myocytes. Biophys J 86(2):1209-1222 
Harris AK, Wild P, Stopak D (1980) Silicone rubber substrata: a new wrinkle in the study of cell locomotion. Science 208(4440):177-179

Heidemann SR, Wirtz D (2004) Towards a regional approach to cell mechanics. Trends Cell Biol 14(4):160-166

Heidemann SR, Kaech S, Buxbaum RE, Matus A (1999) Direct observations of the mechanical behaviors of the cytoskeleton in living fibroblasts. J Cell Biol 145(1):109-122

Hubmayr RD, Shore SA, Fredberg JJ, Planus E, Panettieri RA, Moller W, Heyder J, Wang N (1996) Pharmacological activation changes stiffness of cultured human airway smooth muscle cells. Am J Physiol 271(5 Pt 1):C1660-C1668

Hughes-Fulford M (2004) Signal transduction and mechanical stress. Sci STKE 2004(249):RE12

Ingber DE (1997) Tensegrity: the architectural basis of cellular mechanotransduction. Annu Rev Physiol 59:575-599

Ingber DE (2000) Opposing views on tensegrity as a structural framework for understanding cell mechanics. J Appl Physiol 89(4):1663-1670

Ingber DE (2003) Tensegrity I. cell structure and hierarchical systems biology. J Cell Sci 116(Pt 7):1157-1173

Janson ME, de Dood ME, Dogterom M (2003) Dynamic instability of microtubules is regulated by force. J Cell Biol 161(6):10291034

Jean M (1999) The non-smooth contact dynamics method. Comput Methods Appl Mech Engng 177:235-257

Jean M (2001) Non-smooth contact dynamics approach of cohesive materials. Philos Trans R Soc Lond A 359:2497-2518

Katoh K, Kano Y, Masuda M, Onishi H, Fujiwara K (1998) Isolation and contraction of the stress fiber. Mol Biol Cell 9(7):1919-1938

Kaverina I, Rottner K, Small JV (1998) Targeting, capture, and stabilization of microtubules at early focal adhesions. J Cell Biol 142(1):181-190

Kojima H, Ishijima A, Yanagida T (1994) Direct measurement of stiffness of single actin filaments with and without tropomyosin by in vitro nanomanipulation. Proc Natl Acad Sci USA 91(26):12962-12966

Kole TP, Tseng Y, Huang L, Katz JL, Wirtz D (2004) Rho kinase regulates the intracellular micromechanical response of adherent cells to rho activation. Mol Biol Cell 15(7):34753484

Kole TP, Tseng Y, Jiang I, Katz JL, Wirtz D (2005) Intracellular mechanics of migrating fibroblasts. Mol Biol Cell 16(1):328338

Kurachi M, Hoshi M, Tashiro H (1995) Buckling of a single microtubule by optical trapping forces: direct measurement of microtubule rigidity. Cell Motil Cytoskeleton 30(3):221228

Laurent VM, Canadas P, Fodil R, Planus E, Asnacios A, Wendling S, and Isabey D (2002) Tensegrity behaviour of cortical and cytosolic cytoskeletal components in twisted living adherent cells. Acta Biotheor 50(4):331-356

Laurent VM, Fodil R, Canadas P, Féréol S, Louis B, Planus E, Isabey D (2003) Partitioning of cortical and deep cytoskeleton responses from transient magnetic bead twisting. Ann Biomed Engng 31(10):1263-1278

Maniotis AJ, Chen CS, Ingber DE (1997) Demonstration of mechanical connections between integrins, cytoskeletal filaments, nucleoplasm that stabilize nuclear structure. Proc Natl Acad Sci USA 94(3):849-854

Marek LF, Kelley RO, Perdue BD (1982) Organization of the cytoskeleton in square fibroblasts. Cell Motil 2(2):115-130

McGarry JG, Prendergast PJ (2004) A three-dimensional finite element model of an adherent eukaryotic cell. Eur Cell Mater 7:27-33; discussion 33-4
Meazzini MC, Toma CD, Schaffer JL, Gray ML, Gerstenfeld LC (1998) Osteoblast cytoskeletal modulation in response to mechanical strain in vitro. J Orthop Res 16(2):170-180

Micoulet A, Spatz JP, Ott A (2005) Mechanical response analysis and power generation by single-cell stretching. Chemphyschem 6(4):663-670

Mooney DJ, Langer R, Ingber DE (1995) Cytoskeletal filament assembly and the control of cell spreading and function by extracellular matrix. J Cell Sci 108(Pt 6):2311-2320

Moreau JJ (1993) New computation methods in granular dynamics. Powder Grains 93:227-232

Petersen NO, McConnaughey WB, Elson EL (1982) Dependence of locally measured cellular deformability on position on the cell, temperature, cytochalasin b. Proc Natl Acad Sci USA 79(17):5327-5331

Pienta KJ, Coffey DS (1991) Cellular harmonic information transfer through a tissue tensegrity-matrix system. Med Hypotheses 34(1):88-95

Planus E, Galiacy S, Matthay M, Laurent V, Gavrilovic J, Murphy G, Clérici C, Isabey D, Lafuma C, d'Ortho MP (1999) Role of collagenase in mediating in vitro alveolar epithelial wound repair. J Cell Sci 112(Pt 2):243-252

Pourati J, Maniotis A, Spiegel D, Schaffer JL, Butler JP, Fredberg JJ, Ingber DE, Stamenovic D, Wang N (1998) Is cytoskeletal tension a major determinant of cell deformability in adherent endothelial cells? Am J Physiol 274(5 Pt 1):C1283-C1289

Satcher RL, Dewey CF (1996) Theoretical estimates of mechanical properties of the endothelial cell cytoskeleton. Biophys J 71(1):109-118

Sato K, Adachi T, Matsuo M, Tomita Y (2005) Quantitative evaluation of threshold fiber strain that induces reorganization of cytoskeletal actin fiber structure in osteoblastic cells. J Biomech 38(9):1895-1901

Smith PG, Deng L, Fredberg JJ, Maksym GN (2003) Mechanical strain increases cell stiffness through cytoskeletal filament reorganization. Am J Physiol Lung Cell Mol Physiol 285(2):L456-L463

Stamenovic D, Coughlin MF (1999) The role of prestress and architecture of the cytoskeleton and deformability of cytoskeletal filaments in mechanics of adherent cells: a quantitative analysis. J Theor Biol 201(1):63-74

Stamenovic D, Fredberg JJ, Wang N, Butler JP, Ingber DE (1996) A microstructural approach to cytoskeletal mechanics based on tensegrity. J Theor Biol 181(2):125-136

Stamenovic D, Mijailovich SM, Tolic-Nørrelykke IM, Chen J, Wang N (2002) Cell prestress. II. contribution of microtubules. Am J Physiol Cell Physiol 282(3):C617-C624

Sultan C, Stamenovic D, Ingber DE (2004) A computational tensegrity model predicts dynamic rheological behaviors in living cells. Ann Biomed Eng 32(4):520-530

Tan JL, Tien J, Pirone DM, Gray DS, Bhadriraju K, Chen CS (2003) Cells lying on a bed of microneedles: an approach to isolate mechanical force. Proc Natl Acad Sci USA 100(4):14841489

Thoumine O, Ott A (1996) Influence of adhesion and cytoskeletal integrity on fibroblast traction. Cell Motil Cytoskeleton 35(3):269-280

Thoumine O, Ott A (1997) Time scale dependent viscoelastic and contractile regimes in fibroblasts probed by microplate manipulation. J Cell Sci 110( Pt 17):2109-2116

Thoumine O, Cardoso O, Meister JJ (1999) Changes in the mechanical properties of fibroblasts during spreading: a micromanipulation study. Eur Biophys J 28(3):222-234

Tseng Y, An KM, Esue O, Wirtz D (2004) The bimodal role of filamin in controlling the architecture and mechanics of f-actin networks. J Biol Chem 279(3):1819-1826 
Volokh KY, Vilnay O, Belsky M (2000) Tensegrity architecture explains linear stiffening and predicts softening of living cells. J Biomech 33(12):1543-1549

Wakatsuki T, Kolodney MS, Zahalak GI, Elson EL (2000) Cell mechanics studied by a reconstituted model tissue. Biophys $\mathrm{J}$ 79(5):2353-2368

Wang N (1998) Mechanical interactions among cytoskeletal filaments. Hypertension 32(1):162-165

Wang N, Ingber DE (1994) Control of cytoskeletal mechanics by extracellular matrix, cell shape, mechanical tension. Biophys J 66(6):2181-2189

Wang N, Stamenovic D (2000) Contribution of intermediate filaments to cell stiffness, stiffening, and growth. Am J Physiol Cell Physiol 279(1):C188-C194

Wang N, Butler JP, Ingber DE (1993) Mechanotransduction across the cell surface and through the cytoskeleton. Science 260(5111):1124-1127

Wang N, Naruse K, Stamenovic D, Fredberg JJ, Mijailovich SM, Tolic-Nørrelykke IM, Polte T, Mannix R, Ingber DE (2001) Mechanical behavior in living cells consistent with the tensegrity model. Proc Natl Acad Sci USA 98(14):7765-7770
Wang N, Tolic-Nørrelykke IM, Chen J, Mijailovich SM, Butler JP, Fredberg JJ, Stamenovic D (2002) Cell prestress. I. stiffness and prestress are closely associated in adherent contractile cells. Am J Physiol Cell Physiol 282(3):C606-C616

Wendling S, Oddou C, Isabey D (1999) Stiffening response of a cellular tensegrity model. J Theor Biol 196(3):309-325

Wendling S, Planus E, Laurent VM, Barbe L, Mary A, Oddou C, Isabey D (2000) Role of cellular tone and microenvironmental conditions on cytoskeleton stiffness assessed by tensegrity model. Eur Phys J Appl Physiol 9:51-62

Wendling S, Canadas P, Chabrand P (2003) Toward a generalised tensegrity model describing the mechanical behaviour of the cytoskeleton structure. Comput Methods Biomech Biomed Engng 6(1):45-52

Zhen Y-Y, Libotte T, Munck M, Noegel AA, Korenbaum E (2002) Nuance, a giant protein connecting the nucleus and actin cytoskeleton. J Cell Sci 115(Pt 15):3207-3222 